\title{
Mecambridine induces potent cytotoxic effects, autophagic cell death and modulation of the mTOR/PI3K/Akt signaling pathway in HSC-3 oral squamous cell carcinoma cells
}

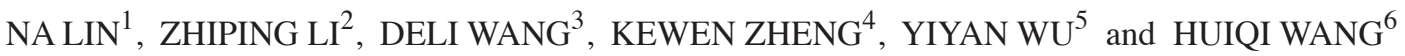 \\ Departments of ${ }^{1}$ Dental and ${ }^{2}$ Pharmacy; ${ }^{3}$ Mudanjiang Medical College; \\ ${ }^{4}$ Department of Orthopeadic Surgery; ${ }^{5}$ College of Pharmacy; ${ }^{6}$ Department of Infectious Disease, \\ Hongqi Hospital of Mudanjiang Medical University, Mudan, Heilongjiang 157011, P.R. China
}

Received March 31, 2017; Accepted August 4, 2017

DOI: $10.3892 / \mathrm{ol} .2017 .7321$

\begin{abstract}
Plant secondary metabolites including alkaloids, demonstrate a complex diversity in their molecular scaffolds and exhibit tremendous pharmacological potential as anti-cancerous therapeutics. The present study aimed to evaluate the anticancer activity of a natural alkaloid, mecambridine, against human oral squamous cell carcinoma (OSCC). An MTT assay was used to evaluate cytotoxic effects of mecambridine on HSC-3 oral squamous cell carcinoma cells. Effects of mecambridine on autophagy-associated proteins were analyzed by western blotting. Effects on reactive oxygen species (ROS) and mitochondrial membrane potential were assessed by flow cytometry. Results indicated that mecambridine exhibited an $\mathrm{IC}_{50}$ value of $50 \mu \mathrm{M}$ and exerted its cytotoxic effects in a dose dependent manner on OSCC HSC-3 cells. Furthermore, it was observed that mecambridine decreases cell viability and induces autophagy in a dose-dependent manner. The underlying mechanism for the induction of autophagy was demonstrated to be associated with ROS-mediated alterations in mitochondrial membrane potential and modulation of the mechanistic target of rapamycin/phosphoinositide 3-kinase/protein kinase $\mathrm{B}$ (m-TOR/PI3K/Akt) signaling pathway in $\mathrm{HSC}-3$ at the $\mathrm{IC}_{50}$. In conclusion, the present study suggests that mecambridine exhibits substantial anticancer activity against OSCC HSC-3 cells by induction of autophagy and modulates the expression of the mTOR/PI3K/Akt signaling cascade which is considered a potential target pathway for anti-cancer agents.
\end{abstract}

Correspondence to: Professor Huiqi Wang, Department of Infectious Disease, Hongqi Hospital of Mudanjiang Medical University, 5 TongXiang Road, Mudan, Heilongjiang 157011, P.R. China

E-mail: huiqiwang12@yahoo.com

Key words: mecambridine, oral squamous cell carcinoma, reactive oxygen species, autophagy

\section{Introduction}

Plants synthesize a wide array of compounds with structurally complex molecular scaffolds. Several of these compounds and their derivatives such as alkaloids and flavonoids exhibit a diversity of medicinal properties (1). Among plant derived secondery metabolites alkaloids are biologically active found across plant kingdom. They have been found to exhibit several pharmacological properties such as anticancer and antimicrobial. Cancer is considered one of the most lethal diseases and due to the dearth of operative drugs, lavish cost of chemotherapeutic agents and the side effects, there is tremendous need for exploration of novel molecules for their anticancer activities (2). Among all cancers, oral squamous cell carcinoma (OSCC) accounts for more 2.5 lakh new cases and about 1.3 lakh deaths each year around the globe (3). If OSCC is detected at an early stage, treatment with surgery or radiotherapy or the combination of both and has a five-year survival rates varying between 70 to $90 \%$ (3-6). However, $2 / 3$ of OSCC patients are diagnosed at advanced stages of the diseases $(6,7)$. In the present study we evaluated the anticancer activity of a plant derived natural alkaloid mecambridine against squamous cell carcinoma HSC-3 oral cell line. Results indicated that mecambridine exhibited an $\mathrm{IC}_{50}$ value of $50 \mu \mathrm{M}$ and exerted its cytotoxic effects in a dose dependent manner on OSCC HSC-3 cell line. Moreover, it was observed that the mecambridine lessens cell viability and induces autophagy dose dependently. The underlying mechanism for the induction of autophagy was found to be ROS mediated in mitochondrial membrane potential and changes in the expression mTOR/PI3K/Akt signalling pathway proteins in HSC-3 at the $\mathrm{IC}_{50}$ concentration of mecambridine. These results strongly stress that mecambridine may prove to be an anticancer lead molecule for the treatment and of OSCC.

\section{Materials and methods}

Chemicals and regents and cell culture conditions. The chemicals used in this study include; triton X-100, dimethyl and sulfoxide (DMSO), RNase A purchased from from Sigma-Aldrich Co., (St. Louis, MO, USA), primary 
and secondary antibodies purchased from Santa Cruz Biotechnology Inc., (Santa Cruz, CA, USA) and fetal bovine serum (FBS), RPMI-1640 medium, L-glutamine, antibiotics procured from Invitrogen Life Technologies (Carlsbad, CA, USA). Squamous cell carcinoma HSC-3 oral cell line was procured from Cancer Research Institute of Beijing, China, and it was maintained in DMEM and was supplemented with $10 \%$ FBS and antibiotics $(100 \mu \mathrm{g} / \mathrm{ml}$ streptomycin and $100 \mathrm{U} / \mathrm{ml}$ penicillin $\mathrm{G})$ in an incubator at $37^{\circ} \mathrm{C}\left(5 \% \mathrm{CO}_{2}\right.$ and $95 \%$ air). Mecambridine was purchased from Chemical Land21 Company, South Korea.

Determination of $I C_{50}$ by MTT assay. The anti-proliferation effect of the mecambridine on cancer cell line squamous cell carcinoma HSC-3 oral cell line was evaluated by MTT assay. HSC-3 cells were grown at $1 \times 10^{6}$ cells per well in 96-well plates for a time period of $12 \mathrm{~h}$ and then exposed to $0,10,25$, $50,100,150$ and $200 \mu \mathrm{M}$ of mecambridine dose for $24 \mathrm{~h}$. To each well, MTT solution ( $20 \mu \mathrm{l}$ of $2.5 \mathrm{mg} / \mathrm{ml}$ stock) was added. Prior to the addition of $500 \mu \mathrm{l}$ of DMSO, the medium was completely removed. To solubilize MTT formazan crystals, $500 \mu 1$ DMSO was added. ELISA plate reader was used for the determination of optical density at $570 \mathrm{~nm}$.

Detection of autophagy. Cells were plates at a density of $1.5 \times 10^{5}$ cells/well treated with either DMSO or $50 \mu \mathrm{M}\left(\mathrm{IC}_{50}\right)$, of mecambridine for $24 \mathrm{~h}$ and successively stained with monodansylcadaverine (MDC) or acridine orange (AO). All cell samples were observed under microscope and Images were captured for at least three independent experiments.

Expression of autophagy related proteins and inhibitor treatment. HSC-3 cells were seeded in 6-well plates at the density of $1.5 \times 10^{5}$ cells/well and kept for $24 \mathrm{~h}$. The cells were then administrated with the mecambridine at $\mathrm{IC}_{50}$ concentration. Untreated cells were included as control. Following $24 \mathrm{~h}$ of treatment, cells were collected and lysed for quantification of proteins and expression analysis. For inhibitor treatment, HSC-3 cells were seeded at the density of $1.5 \times 10^{5}$ cells/well in 6-well plates and permitted to adhere for $24 \mathrm{~h}$. Cells were then administrated with $15 \mu \mathrm{g} / \mathrm{ml}$ of the liposomal inhibitor pepstatin A for $1 \mathrm{~h}$ with and then administrated $\mathrm{IC}_{50}$ concentration of mecambridine for $24 \mathrm{~h}$. untreated cells were kept as control. Protein expression analysis was carried out by Western blot analysis.

Evaluation of ROS and MMP. HSC-3 cells were platted at a density of $2 \times 10^{5}$ cells/well in a 6 -well plate and kept for $24 \mathrm{~h}$ and treated with $0,25,50$ and $100 \mu \mathrm{M}$ mecambridine for $72 \mathrm{~h}$ at $37^{\circ} \mathrm{C}$ in $5 \% \mathrm{CO}_{2}$ and $95 \%$ air. Thereafter cells from all samples were collected, washed 2 times by PBS and re-suspended in $500 \mu \mathrm{l}$ of DCFH-DA $(10 \mu \mathrm{M})$ for ROS estimation and $\mathrm{DiOC}_{6}$ (1 $\mu \mathrm{mol} / \mathrm{l}$ ) for MMP at $37^{\circ} \mathrm{C}$ in dark room for $30 \mathrm{~min}$. The samples were then examined instantly using flow cytometer as described previously in literature (8).

Protien expression by western blot analysis. The mecambridine administrated cells were harvested and lysed. The protein concentrations of the lysates were quantified by BCA assay using specific antibodies. $\beta$-actin was used as a control. From each sample equal amounts of protein were loaded and

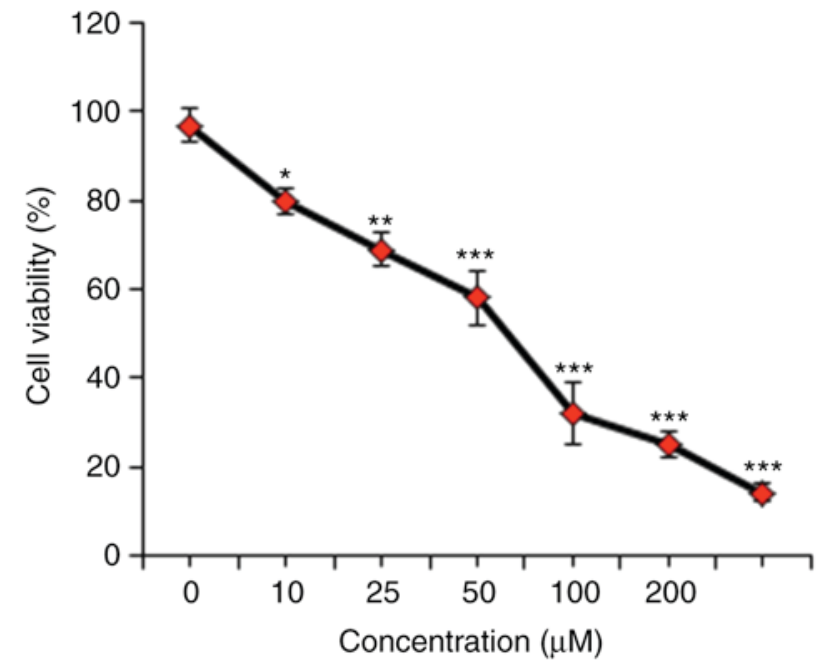

Figure 1. Effect of indicated doses of mecambridine cell viability. All experiments were carried out in triplicates and represent mean \pm SD. Results were considered significant at ${ }^{*} \mathrm{P}<0.01,{ }^{* *} \mathrm{P}<0.001,{ }^{* * *} \mathrm{P}<0.0001$.

separated by electrophoresis on a $12 \%$ denaturing SDS gel. Afterwards, the proteins were electroblotted on polyvinylidene difluoride membranes ( $0.45 \mathrm{~m}$ pore size).

Statistical analysis. All experiments were carried out in triplicates and presented as representative images or average values $\pm \mathrm{SD}$. Results were considered significant at ${ }^{*} \mathrm{P}<0.01$, ${ }^{* *} \mathrm{P}<0.001,{ }^{* * *} \mathrm{P}<0.0001$.

\section{Results}

Cytotoxic potential of mecambridineon HSC-3 cell line. The growth inhibitory role of mecambridine on HSC-3 cells was detected by treatment of these cells with varied concentrations of mecambridine. Mecambridine displayed the potent anti-proliferative effect against $\mathrm{HSC}-3$ cells with an $\mathrm{IC}_{50}$ of $50 \mu \mathrm{M}$ (Fig. 1). The anti-proliferative activity of the mecambridine was found be concentration-dependent.

Mecambridine induces autophagy in HSC-3 cells. In order to confirm that mecambridine induces autophagy in HSC-3 cells, mecambridine treated cells were stained with MDC. Vital staining of mecambridine-treated HSC-3 cells with MDC (monodansylcadaverine, an autophagolysosome marker) and AO (acridine orange), indicated an increased buildup of the dye as compared to the control cells. In control the dye is scattered and comparatively fainter than treatment (Fig. 2). These observations provided strong clue that mecambridine induces autophagy in HSC-3 cells. To quantify the increase of the acidic vesicular organelles, mecambridine-treated cells were treated with acridine orange dye and it was revealed that there was accumulation of acridine orange in the mecambridine-treated cells.

To confirm autophagy, we evaluated the expression of several autophagy associated proteins. The results indicated that the treatment with the extract induced the expression of several autophagy associated proteins (Fig. 3). It was observed there was no change in the expression of several proteins which include Vps34, Beclin-1, and LC3-I. However expression 

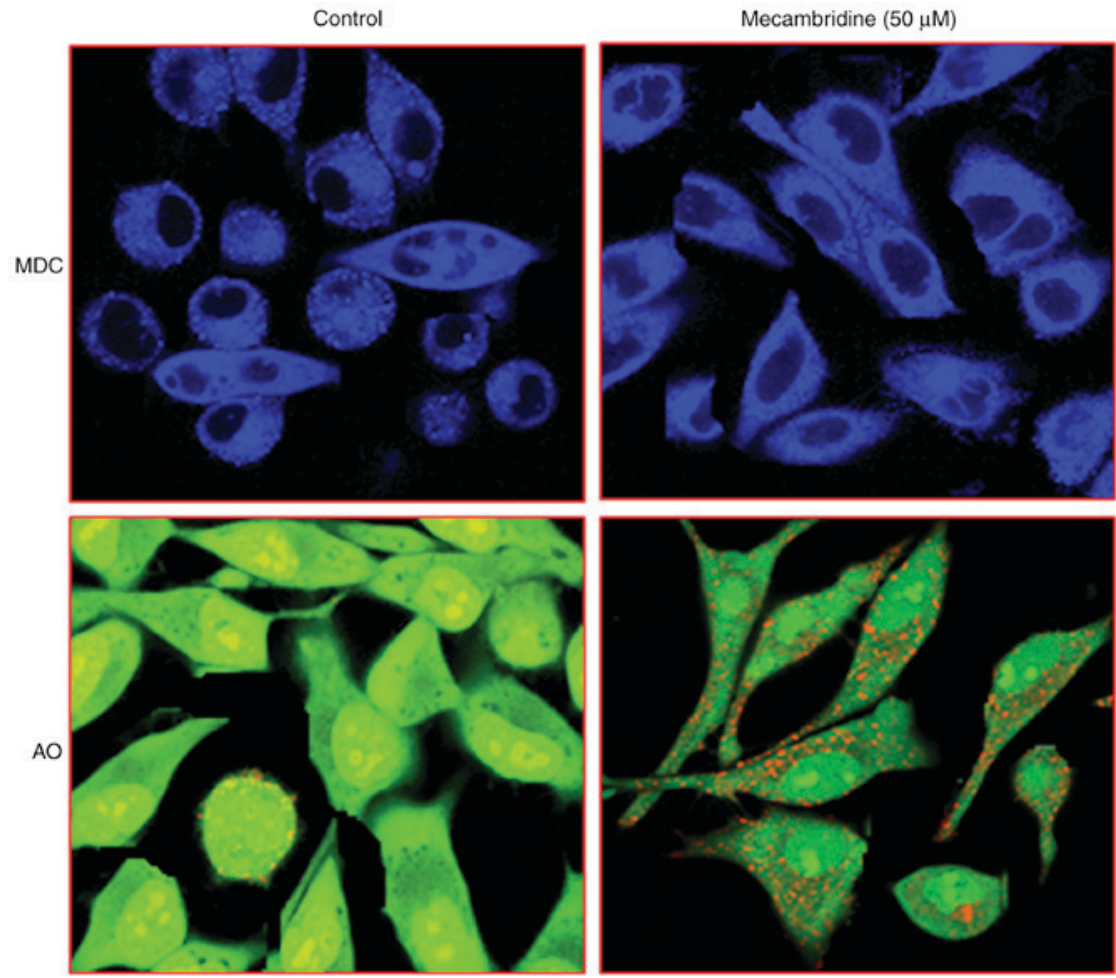

Figure 2. Induction of autophagy by mecambridine at $\mathrm{IC}_{50}$ concentration. The cells were stained with $\mathrm{MDC}$ and $\mathrm{AO}$ and observed under fluorescence microscope (200X). All experiments are representatives of three biological replicates. Blue color indicates autophagosomes, green color indicates absence of autophagic vesicles, and red color indicates autophagic vesicles. MDC, monodansylcadaverine; AO, acridine orange.

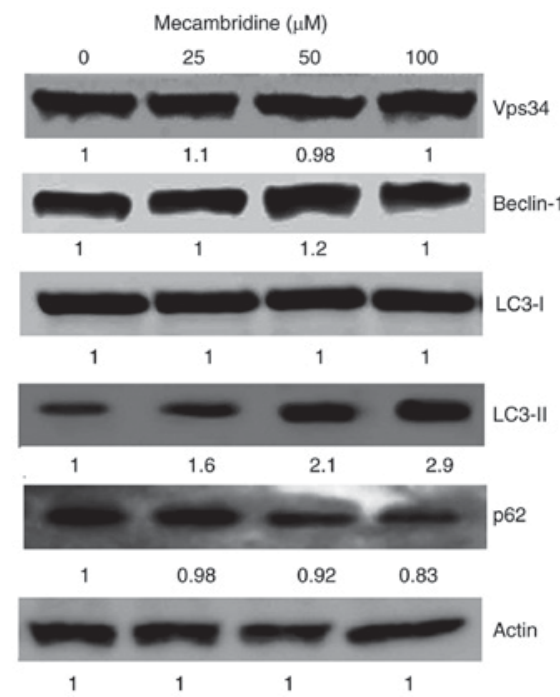

Figure 3. Expression pattern of autophagy associated proteins at indicated concentrations of mecambridine by western blotting. All experiments are representative of three biological replicates.

of LC3-II was significantly increased in a concentration dependent manner while as slight reduction in the expression of together with a slight reduction in the levels of p62 was also observed. The capacity of the mecambridine to induce autophagy was further confirmed by the use of autophagy inhibitor, pepstatin. The results indicated that mecambridine abridged the effect of the inhibitors (Fig. 4).

Mecambridine induces the ROS accretions andMMPreduction in HSC-3 cells. The autophagic potential of mecambridine

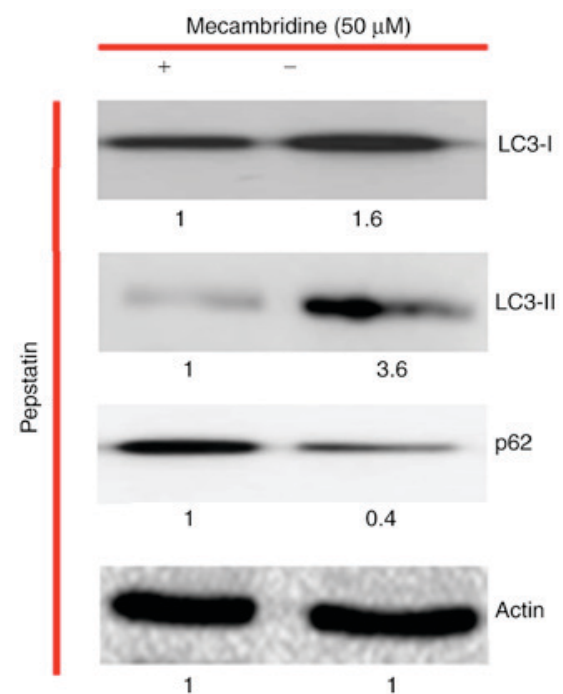

Figure 4. Mecambridine abolishes the effect of autophagy inhibitors at $\mathrm{IC}_{50}$ concentrations. All experiments are representative of three biological replicates. MDC, monodansylcadaverine; AO, acridine orange.

indicated that it might induce generation of intracellular ROS. Therefore, we calculated the ROS level at varied concentrations of mecambridine for $24 \mathrm{~h}$. The results showed that the intracellular ROS levels of treated cells increased to $194 \%$ at $100 \mu \mathrm{M}$ as compared to untreated cells (Fig. 5). Our result suggested that mecambridine a potent molecule for activating ROS in squamous oral carcinoma HSC-3 cells to trigger the autophagy. ROS generation causes mitochondrial dysfunction. It disrupts the outer mitochondrial potential to release the death-promoting proteins (9). Therefore, we examined whether 


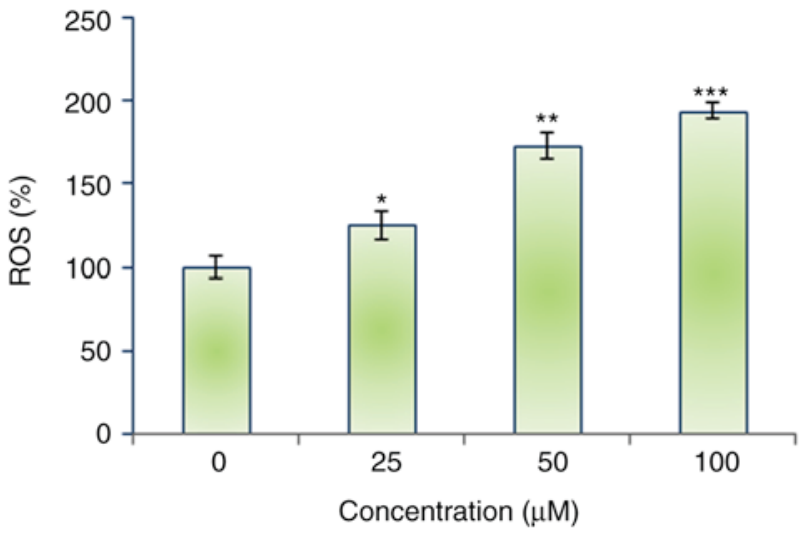

Figure 5. Effect of indicated concentration of mecambridineon Reactive oxygen species production. All experiments are replicates of three biological experiments \pm SD. Results were considered significant at ${ }^{*} \mathrm{P}<0.01,{ }^{* *} \mathrm{P}<0.001$, **** $\mathrm{P}<0.0001$.

mecambridine reduces the MMP in squamous oral carcinoma HSC-3 cells in a concentration dependent manner. Treated squamous oral carcinoma HSC-3 cells showed a significant reduction in MMP in a dose-dependent manner. The MMP reduced by $68 \%$ at $100 \mu \mathrm{M}$ of mecambridine as compared to untreated control (Fig. 6).

Mecambridine targets $m$-TOR/PI3K/Akt signalling pathway. The fact that mecambridine could modulate the protein expressions of m-TOR/PI3K/Akt signalling pathway was evaluated by using western blot analysis (Fig. 7). Compared to the untreated control cells, mecambridine treated HSC-3 cells showed a concentration-dependent downregulation of m-TOR and pm-TOR proteins. It also caused downregulation of PI3K/Akt protein expressions. Thus it may be concluded that mecambridine induced anticancer and autophagy inducing effects via m-TOR/PI3K/Akt signalling pathway.

\section{Discussion}

Oral squamous cell carcinoma is of the leading causes of cancer related mortality and about 1.3 lakh new patients are diagnosed for OSCC every year across the world. Additionally, treatment options for this type of cancer are limited and currently available treatments have severe side effects and badly affects the quality line of the patients $(4,5)$. Therefore, the present study aimed at determining the anticancer activity of the mecambridine (a natural alkaloid) against OSCC HSC-3 cells. The results indicated that the test molecule mecambridine exerted significant anticancer activity against OSCC HSC-3 cells in a dose dependent manner with an $\mathrm{IC}_{50}$ of $50 \mu \mathrm{M}$. These results suggest that the mecambridine is a potential source of cytotoxic agents. The cytotoxic effect of mecambridine was later on reported to be due to the induction of autophagy, as evident from the accumulation of MDC and acridine orange dyes in mecambridine treated OSCC HSC-3 cells. Expression of several of the autophagy associated proteins was evaluated and it was found that the expression of only LC3-II was highly induced by the mecambridine in OSCC HSC-3 cells. Furthermore mecambridine exhibited a strong potential to abridge the expression of autophagy inhibitors, providing a

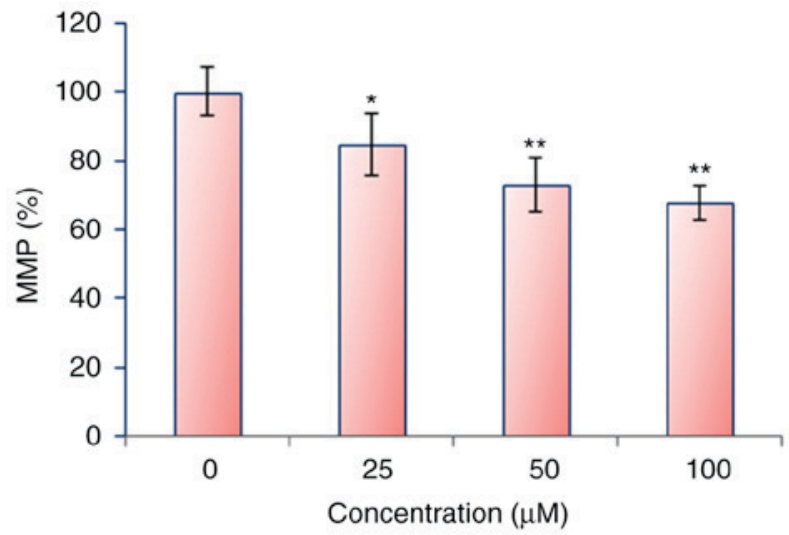

Figure 6. Effect of indicated concentration of mecambridine on mitochondrial membrane potential. All experiments are replicates of three biological experiments \pm SD. Results were considered significant at ${ }^{*} \mathrm{P}<0.01,{ }^{* *} \mathrm{P}<0.001$

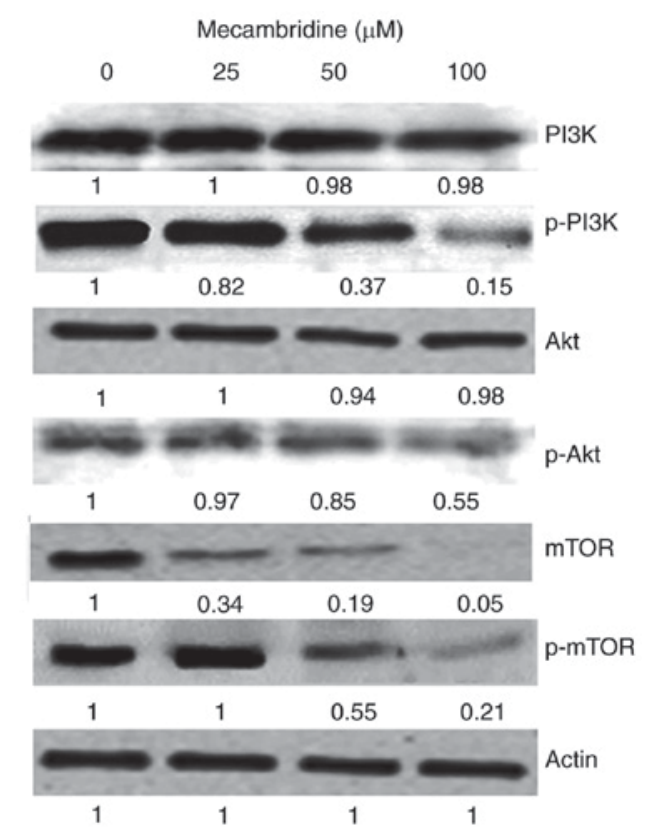

Figure 7. Effect of indicated concentrations of mecambridine on the protien expression levels of mTOR/PI3/Akt pathway. Experiments are representative of three biological replicates.

strong clue towards the role of this molecule in the execution of autophagy.

Moreover, results indicated that mecambridine treated cells displayed ROS mediated MMP reduction. Therefore, the results suggest that the mecambridine may induce autophagy through increasing intracellular ROS and reduction in MMP. Our results are in agreement with studies wherein several anti-tumor agents have been reported to target cancer cells partly by accretion of high levels of ROS (10-15). Finally, effects of mecambridine on the expression levels of various proteins including m-TOR, pm-TOR, PI3K, p-PI3K and Akt were studied using western blot assay. Results showed mecambridine-treated OSCC HSC-3 cells showed a concentration-dependent downregulation of $\mathrm{m}$-TOR and pm-TOR proteins. It also caused downregulation of PI3K/Akt protein expressions. Therefore, inhibitory effect of mecambridineon 
OSCC HSC-3 cells may prove crucial in the treatment and management of OSCC.

In conclusion, the present results suggest that mecambridine induces anticancer and autophagy effects via the m-TOR/PI3K/Akt signalling pathway. The mechanism involved will be further studies in the future.

\section{Acknowledgements}

The study was supported by Science and Technology Plan Project of Mudanjiang (grant no. Z2016s0077).

\section{References}

1. McChesney JD, Venkataraman SK and Henri JT: Plant natural products: Back to the future or into extinction? Phytochem 68 2015-2022, 2007.

2. George S, Bhalerao SV, Lidstone EA, Ahmad IS, Abbasi A Cunningham BT and Watkin KL: Cytotoxicity screening of Bangladeshi medicinal plant extracts on pancreatic cancer cells. BMC Complement Altern Med 10: 52, 2010.

3. Ferlay J, Soerjomataram I, Ervik M, Dikshit R, Eser S, Mathers C, Rebelo M, Parkin DM, Forman D and Bray F: Cancer Incidence and Mortality Worldwide: IARC Cancer Base No. 11 [Internet] Lyon, France: International Agency for Research on Cancer; 2013: GLOBOCAN 2012 v1.0, 2012. Available from: http://globocan.iarc.fr.

4. Scully C and Bagan JV: Recent advances in Oral Oncology 2007 Imaging, treatment and treatment outcomes. Oral Oncol 44 211-215, 2008.

5. Argiris A, Karamouzis MV, Raben D and Ferris RL: Head and neck cancer. Lancet 371: 1695-1709, 2008.
6. Nagao T, Chaturvedi P, Shaha A and Sankaranarayanan R: Prevention and early detection of head and neck squamous cell cancers. J Oncol 2011: 318145, 2011.

7. Rousseau A and Badoual C: Head and Neck: Squamous cell carcinoma: An overview. Atlas Gen Cytogenetics Oncol Haematol 16: 145-155, 2012.

8. Hissin PJ and Hilf R: A fluorometric method for determination of oxidized and reduced glutathione in tissues. Anal Biochem 74: 214-226, 1976

9. Azuma M, Tamatani T, Ashida Y, Takashima R, Harada K and Sato M: Cisplatin induces apoptosis in oral squamous carcinoma cells by the mitochondria-mediated but not theNF-kappaB-suppressed pathway. Oral Oncol 39: 282-289, 2003.

10. Shoemaker RH: The NCI60 human tumour cell line anticancer drug screen. Nat Rev Cancer 6: 813-823, 2006

11. Sreelatha S, Jeyachitra A and Padma PR: Antiproliferation and induction of apoptosis by Moringa oleifera leaf extract on human cancer cells. Food Chem Toxicol 49: 1270-1275, 2011.

12. Rejiya CS, Cibin TR and Abraham A: Leaves of Cassia tora as a novel cancer therapeutic-An in vitro study. Toxicol In Vitro 23: 1034-1038, 2009.

13. Ding H, Han C, Guo D, Chin YW, Ding Y, Kinghorn AD and D'Ambrosio SM: Selective induction of apoptosis of human oral cancer cell lines by avocado extracts via a ROS mediated mechanism. Nutr Cancer 61: 348-356, 2009.

14. Kowaltowski AJ, de Souza-Pinto NC, Castilho RF and Vercesi AE: Mitochondria and reactive oxygen species. Free Radic Biol Med 47: 333-343, 2009.

15. Aadil K, Manzoor AR and Rafiya R: Plant-based natural compounds and herbal extracts as promising apoptotic agents: Their implications for cancer prevention and treatment. Advan Biomed Pharma 3: 245-269, 2016. International (CC BY-NC-ND 4.0) License. 